\title{
On expansive chaotic maps in G-spaces
}

\author{
Salah H. Abid ${ }^{1 *}$, Ihsan J. Kadhim ${ }^{2}$ \\ ${ }^{1}$ Mathematics department, Education college, Al-Mustansiriya University, Baghdad, Iraq \\ ${ }^{2}$ Department of Mathematics, College of Computer Science and Mathematics, University of Al-Qadysia \\ *Corresponding author E-mail: abidsalah@gmail.com
}

Copyright $\odot 2014$ Salah H. Abid, Ihsan J. Kadhim This is an open access article distributed under the Creative Commons Attribution License, which permits unrestricted use, distribution, and reproduction in any medium, provided the original work is properly cited.

\begin{abstract}
In this paper we define expansive maps in general topological space (not necessarily metrizable space) and generalize this definition to G-spaces and give some propertie of such maps. Also we study some properties of new type of chaotic maps which we called G-expansive chaotic maps.
\end{abstract}

Keywords: G-Periodic Points, G-Orbits, G-Expansive Maps, G-Expansive Chaos.

\section{Introduction}

One of the important dynamical properties studied for dynamical systems is expansive which was introduced by Utz [18] in 1950 for homeomorphisms on metric spaces. Expansive homeomorphisms have lots of applications in Topological Dynamic, Ergodic Theory, Continuum Theory, Symbolic Dynamics etc. With the intention of studying various dynamical properties of maps under the continuous action of a topological group, the notation of expansiveness termed as G-expansive homeomorphism was defined for a self-homeomorphism on a metric G - space [4]. It is observed that the notion of expansiveness and the notation of $G$-expansive under a non trivial action of $G$ are independent of each other. Conditions under which an expansive homeomorphism on a metric $G-$ space is $\mathrm{G}$ - expansive and vice-versa are also obtained. The same paper also contains a characterization of $\mathrm{G}$ - expansive homeomorphism and condition under which it can be extended from a subspace to the whole space. In the process of above study a useful notion of pseudo equivariant map is defined. Recently Choi and Kim in [3] have used this concept to generalize topological decomposition theorem proved in [1] due to Aoki and Hiraide for compact metric G - spaces. Further, in [5] the notation of generators in G-spaces termed as G-generator is defined and a characterization of $\mathrm{G}$-expansive homeomorphism is obtained using $\mathrm{G}$ - generator. Some interesting consequences have been obtained for example it is shown that an arc cannot admit a pseudo equivariant $\mathrm{G}$-expansive homeomorphism. It is well known that an arc does not admit an expansive homeomorphism .R.Das and T.K.Das in [10] studied the extension of G - expansive homeomorphism. R.Das and T. Das in [9] studied some properties of G -expansive homeomorphism. It is worth noting here that other dynamical properties like shadowing and transitivity of maps on G -space are also studied [6], [17].

Throughout $\mathcal{H}(\mathrm{X})$ denote the collection of all self-homeomorphisms of a topological space, $\mathbb{R}$ denotes the set of all real numbers, $\mathbb{Z}$ denotes the set of integers and $\mathbb{N}$ denotes the set of positive integers. By a $G$-space (or transformation group) [2], [12], [13] we mean a triple $(X, G, \theta)$, where $X$ is a topological space, $G$ is a topological group and $\theta: G \times X \rightarrow$ $X$ is a continuous action of $G$ on $X$. Forx $\in X$, the set $G(x)=\{\theta(g, x): g \in G\}$, is called the $G$-orbit of $x$ in $X$. Note that $G$ - orbits $G(x)$ and $G(y)$ of points $x, y \in X$ are either disjoint or equal. A subset $S$ of $X$ is called $G-$ invariant if $\theta(G \times$ $S) \subseteq S$. Let $X / G=\{G(x): x \in X\}$ and $p_{X}: X \rightarrow X / G$ be the natural quotient map taking $x$ to $(x)$, then $X / G$ endowed with the quotient topology is called the orbit space of $X$ (with respect to $G$ ). The map $p_{X}$ is also a closed map. An action of $G$ on $\mathrm{X}$ is called transitive if $\mathrm{X} / \mathrm{G}=\{\mathrm{X}\}$. If $\mathrm{X}, \mathrm{Y}$ are $\mathrm{G}$ - spaces, then a continuous map $\mathrm{h}: \mathrm{X} \rightarrow \mathrm{Y}$ is called equivariant if $\mathrm{h}(\theta(\mathrm{g}, \mathrm{x}))=\theta(\mathrm{g}, \mathrm{h}(\mathrm{x}))$ for each $\mathrm{g}$ in $\mathrm{G}$ and each xin. We call h pseudoequivariant if $\mathrm{h}(\mathrm{G}(\mathrm{x}))=\mathrm{G}(\mathrm{h}(\mathrm{x}))$ for each $\mathrm{xin}$. An equivariant map is clearly pseudoequivariant but converse is not true [4].

By a metric $G$ - space, we mean a metric space on which a topological group $\mathrm{G}$ acts.

Recall that if $\mathrm{X}$ is a metric space with metric $\mathrm{d}$ and $\mathrm{h} \in \mathcal{H}(\mathrm{X})$ then $\mathrm{h}$ is called expansive[18], if there exists a $\delta>0$ such that whenever $\mathrm{x}, \mathrm{y} \in \mathrm{X}, \mathrm{x} \neq \mathrm{y}$ then there exists an integer $\mathrm{n}$ satisfying $\mathrm{d}\left(\mathrm{h}^{\mathrm{n}}(\mathrm{x}), \mathrm{h}^{\mathrm{n}}(\mathrm{y})\right)>\delta$; $\delta$ is then called an 
expansive constant for $h$. A topological space $X$ is said to be Urysohn space $\left(T_{2}\right)$ [19]if for every pair of distinct points $\mathrm{x}, \mathrm{y} \in \mathrm{X}$ there exist pair of disjoint open sets $\mathrm{U}$ and $\mathrm{V}$ such that $\mathrm{x} \subset \mathrm{U}, \mathrm{y} \subset \mathrm{V}$ and $\overline{\mathrm{U}} \cap \overline{\mathrm{V}}=\phi($ respectively $\mathrm{U} \cap \mathrm{V}=\emptyset$ ). Every Urysohn space is $T_{2}$ but the converse need not be true. A topological space $X$ is said to be $T_{1}[19]$ if for every pair of distinct points $\mathrm{x}, \mathrm{y} \in \mathrm{X}$ there exist pair of open sets $\mathrm{U}$ and $\mathrm{V}$ such that $\mathrm{x} \subset \mathrm{U}, \mathrm{y} \subset \mathrm{V}$.Thus $\mathrm{X}$ is $\mathrm{T}_{1}$ iff every singleton set in $X$ is closed [19]. A topological space $X$ is said to be normal [19]if for every pair of disjoint closed sets $F_{1}, F_{2} \subset X$ there exist pair of open sets $U$ and $V$ such that $F_{1} \subset U, F_{2} \subset V$ and $U \cap V=\phi$. A topological space $X$ is normal [19]iff for every pair of disjoint closed sets $F_{1}, F_{2} \subset X$ there exist pair of open sets $U$ and $V$ such that $F_{1} \subset U, F_{2} \subset V$ and $\bar{U} \cap \bar{V}=\emptyset$. A topological space $X$ is said to be compact if every open cover of $X$ admits a finite subcover. A compact $T_{2}$ space is normal [19]. A topological group $G$ is said to be compact [19] if it is compact as a topological space.

In Section 2 we state the definition of $G$ - periodic point and prove sum properties of such points. In Section 3, we state the definition of $\mathrm{G}$-orbit of maps and give some new properties of the $\mathrm{G}$-orbits of maps. In Section 4, we introduce the concept of expansive maps in $\mathrm{G}$ - space and obtain the necessarly condition auder which the map becomes expansive. Further we study some important properties required in Section 5. In Section 5, we introduce a new type of chaotic maps called G - expansive chaotic map and show that there are no redundant hypotheses in our definition of chaos. Also we study some properties of these maps. In Section 6, we give some examples of maps that are $\mathrm{G}$-expansive chaotic or not $\mathrm{G}$-expansive chaotic.

Definition1.1: [12] The pair of maps $(\mu, \psi):\left(G_{1}, X, \theta_{1}\right) \rightarrow\left(G_{2}, Y, \theta_{2}\right)$ is said to be homeomorphism between the two transformation groups $\left(G_{1}, X, \theta_{1}\right)$ and $\left(G_{2}, Y, \theta_{2}\right)$ if

i) $\quad \mu: G_{1} \rightarrow G_{2}$ is topological group homomorphism,

ii) $\quad \psi: \mathrm{X} \rightarrow \mathrm{Yis}$ continuous map and

iii) $\psi\left(\theta_{1}(\mathrm{~g}, \mathrm{x})\right)=\theta_{2}(\mu(\mathrm{g}), \psi(\mathrm{x}))$.

Definition 1.2: [12] the homeomorphism $(\mu, \psi)$ between two transformations groups $\left(G_{1}, X, \theta_{1}\right)$ and $\left(G_{2}, Y, \theta_{2}\right)$ is said to be isomorphism if $\mu$ is topological group isomorphism and $\psi$ is homeomorphism. If there exists such map between $\left(G_{1}, X, \theta_{1}\right)$ and $\left(G_{2}, Y, \theta_{2}\right)$ we say that they are isomorphic and we write $\left(G_{1}, X, \theta_{1}\right) \cong_{(\mu, \psi)}\left(G_{2}, Y, \theta_{2}\right)$.

Note that in this case $(\mu, \psi)^{-1}:=\left(\mu^{-1}, \psi^{-1}\right)$.

Definition 1.3: Let $\left(G_{1}, X, \theta_{1}\right)$ and $\left(G_{2}, Y, \theta_{2}\right)$ be two transformation groups. Two maps $f: X \rightarrow X$ and h: $Y \rightarrow Y$ are said to be equivariant topologically conjugate if there exists an isomorphism $(\mu, \psi):\left(G_{1}, X, \theta_{1}\right) \rightarrow\left(G_{2}, Y, \theta_{2}\right)$ such that $\psi$ is topological conjugate, i.e., $\psi \mathrm{f}=\mathrm{h} \psi$. In this case we say that $\mathrm{f}: \mathrm{X} \rightarrow \mathrm{X}$ and $\mathrm{h}: \mathrm{Y} \rightarrow \mathrm{Y}$ are equivariant topologically conjugate via

$(\mu, \psi):\left(G_{1}, X, \theta_{1}\right) \rightarrow\left(G_{2}, Y, \theta_{2}\right)$.

Example 1.4: Let $X=Y=[0,1]$ with the usual topology, and let $\mathbb{Z}_{2}$ act on $X$ by $\theta(0, x)=x a n d \theta(1, x)=1-x, x \in X$. Let $\mathrm{Q}_{4}: \mathrm{X} \rightarrow \mathrm{X}$ be the logistic map defined by $\mathrm{Q}_{4}(\mathrm{x})=4 \mathrm{x}(1-\mathrm{x})$ and let $\mathrm{T}: \mathrm{X} \rightarrow \mathrm{X}$ be the Tent map defined byT $(\mathrm{x})=$ $1-|2 x-1|$. Then $T$ and $Q_{4}$ are equivariant topologically conjugate $\operatorname{via}(\mu, \psi):(G, X, \theta) \rightarrow(G, X, \theta)$, where $\mu: G \rightarrow G$ be the identity map and the map $\psi: X \rightarrow X$ defined by $\psi(x)=\left(\sin \frac{\pi}{2} x\right)^{2}$.

\section{G-periodic points}

In this section we state the definition of periodic and $\mathrm{G}$ - periodic points. Also we give some properties related with the $\mathrm{G}$ - periodic points.

Definition 2.1: [11] Let $X$ be a topological space and $f: X \rightarrow X$ be a map. A point $x \in X$ is said to be periodic point of $f$ if there exist $\mathrm{n} \in \mathbb{N}_{\text {such }}$ thatf $^{\mathrm{n}}(\mathrm{x})=\mathrm{x}$. The smallest positive integer $\mathrm{n}$ satisfy the equationf ${ }^{\mathrm{n}}(\mathrm{x})=\mathrm{x}$ is called periodic of f.

Definition 2.2: [7] Let $X$ be a $G$-space and $f: X \rightarrow X$ be a map. A point $x \in X$ is said to be $G$-periodic point of $f$ if there exist $n \in \mathbb{N}$ and $g \in G \operatorname{such}_{\operatorname{tatf}^{n}}(x)=\theta(g, x)$. The smallest positive integer $n$ satisfy the equationf ${ }^{n}(x)=\theta(g, x)$ is called periodic off.

Remark 2.3: Under trivial action of GonX, notation of periodic point and G - periodic point coincide. Under non-trivial action of GonX, if $x \in X$ is periodic point of $f: X \rightarrow X$, then it is $G$-periodic point. But the converse need not be true as we see in the following example.

Example 2.4.Let $X=[0,1]$ under the usual topology, $G=\{-1,1\}$ under the discrete topology. Define $\theta: G \times X \rightarrow X$ by $\theta(-1, x)=1-x$ and $\theta(1, x)=x, x \in X$. Then $\theta$ is a continuous action of GonX. Let $T: X \rightarrow X$ be the tent map. Then $\mathrm{x}=\frac{1}{7}$ is $\mathrm{G}$ - peridic point but not periodic point. 
Theorem 2.5: Let $\left(X, G_{1}, \theta_{1}\right)$ be $G_{1}$-space and $\left(Y, G_{2}, \theta_{2}\right)$ be $G_{2}$ - space. Let $f: X \rightarrow X$ and $h: Y \rightarrow Y$ be equivariant maps. Then $x \in X$ is $G_{1}$-periodic point of $f$ and $x \in Y$ is $G_{2}$-periodic point of $h$ if and only if $(x, y) \in X \times Y$ is $G_{1} \times G_{2}-$ periodic point off $\times h$.

Proof: See the proof of Theorem 3.2 in [7].

Corollary 2.6. Let $\left(X, G_{1}, \theta_{1}\right)$ be $G_{1}-$ space and $\left(Y, G_{2}, \theta_{2}\right)$ be $G_{2}-$ space. Let $f: X \rightarrow X$ and $h: Y \rightarrow Y$ be equivariant maps. If $x \in X$ is $G_{1}$-periodic point of $f$ of period $n_{1}$ and $x \in Y$ is $G_{2}$-periodic point of $h$ of period $n_{2}$ then $(x, y) \in X \times$ $Y$ is $G_{1} \times G_{2}$ - periodic point of $f \times h$ with period $n=\operatorname{lcm}\left(n_{1}, n_{2}\right)$.

Theorem 2.7: Let $\left(X, G_{1}, \theta_{1}\right)$ be $G_{1}-$ space and $\left(Y, G_{2}, \theta_{2}\right)$ be $G_{2}-$ space. Let $f: X \rightarrow X$ and $h: Y \rightarrow Y$ be equivariant maps. If the set $G_{1}$-periodic point of $f$ is dense in $X$ and the set of $G_{2}$-periodic point of $h$ is dense in $Y$ then the set of $G_{1} \times G_{2}$-periodic point of $f \times h$ is dense in $X \times Y$.

Proof: See the proof of Theorem 3.2 in [7].

Theorem 2.8: Let $\left(G_{1}, X, \theta_{1}\right),\left(G_{2}, Y, \theta_{2}\right)$ be two transformation groups and the two maps $f: X \rightarrow X, h: Y \rightarrow Y$ are to be equivariant topologically conjugate via $(\mu, \psi):\left(G_{1}, X, \theta_{1}\right) \rightarrow\left(G_{2}, Y, \theta_{2}\right)$. If $x_{0} \in X$ is a $G_{1}$-periodic point of $f$, then $\varphi\left(x_{0}\right)$ is a $G_{2}$-periodic point of $h$.

Proof: Suppose that $f$ and $h$ are equvalent topologically conjugate via $\varphi: X \rightarrow Y$. If $x \in X$ is a $G_{1}$-periodic point of , then there exists $n \in \mathbb{N}$ and $g_{1} \in G_{1}$ such that $\mathrm{f}^{\mathrm{n}}\left(\mathrm{x}_{0}\right)=\theta_{1}\left(\mathrm{~g}_{1}, \mathrm{x}_{0}\right)$. Thus $\varphi\left(\mathrm{f}^{\mathrm{n}}\left(\mathrm{x}_{0}\right)\right)=\varphi\left(\theta_{1}\left(\mathrm{~g}_{1}, \mathrm{x}_{0}\right)\right)$, for some $\mathrm{n} \in \mathbb{N}$ and $\mathrm{g}_{1} \in \mathrm{G}_{1}$. Since $\varphi$ equavaliant, then

$\varphi\left(\theta_{1}\left(\mathrm{~g}_{1}, \mathrm{x}\right)\right)=\theta_{2}\left(\mu\left(\mathrm{g}_{1}\right), \varphi(\mathrm{x})\right)$, for every $\mathrm{g}_{1} \in \mathrm{G}_{1}$, and $\mathrm{x} \in \mathrm{X}$.

$\operatorname{Thus} \varphi\left(\theta_{1}\left(\mathrm{~g}_{1}, \mathrm{x}_{0}\right)\right)=\theta_{2}\left(\mu\left(\mathrm{g}_{1}\right), \varphi\left(\mathrm{x}_{0}\right)\right)$, for some $\mathrm{g}_{1} \in \mathrm{G}_{1}$.

Hence $\varphi\left(\mathrm{f}^{\mathrm{n}}\left(\mathrm{x}_{0}\right)\right)=\theta_{2}\left(\mu\left(\mathrm{g}_{1}\right), \varphi\left(\mathrm{x}_{0}\right)\right)$. Now, since $\varphi$ is topological conjugacy, then $\varphi \mathrm{f}^{\mathrm{n}}=\mathrm{h}^{\mathrm{n}} \varphi$. Therefore $\mathrm{h}^{\mathrm{n}}\left(\varphi\left(\mathrm{x}_{0}\right)\right)=\theta_{2}\left(\mathrm{~g}_{2}, \varphi\left(\mathrm{x}_{0}\right)\right)$, where $\mu\left(\mathrm{g}_{1}\right)=\mathrm{g}_{2} \in \mathrm{G}_{2}$. That is $\varphi\left(\mathrm{x}_{0}\right)$ is a $\mathrm{G}_{2}$-periodic ponit of $\mathrm{h}$ and this complete the proof.

Notation. We denote $\mathrm{K}(\mathrm{X})$ the set of all compact subset in $\mathrm{X}$,i.e, $\mathrm{K}(\mathrm{X})=\{\mathrm{F} \subset \mathrm{X}$ : $\mathrm{F}$ is compact set in $\mathrm{X}\}$.

Theorem 2.9: Let $\left(G_{1}, X, \theta_{1}\right),\left(G_{2}, Y, \theta_{2}\right)$ be two transformation groups and $f: X \rightarrow X, h: Y \rightarrow Y$ be equivariant topologically conjugate via $(\mu, \psi):\left(G_{1}, X, \theta_{1}\right) \rightarrow\left(G_{2}, Y, \theta_{2}\right)$. Let $F \in K(X)$. If $\overline{\operatorname{GPr}(f, F)}=F$, then $\overline{\operatorname{GPr}(h, \psi(F))}=$ $\psi(F)$.

Proof: First note that if $\mathrm{F} \in \mathrm{K}(\mathrm{X})$, then $\psi(\mathrm{F}) \in \mathrm{K}(\mathrm{Y})$. Suppose, if possible, that $\overline{\mathrm{GPr}(\mathrm{h}, \psi(\mathrm{F}))} \neq \psi(\mathrm{F})$. Then there is an open set $V$ in $\psi(F)$ such that $G \operatorname{Pr}(h, \psi(F)) \cap V=\phi$. Let $y \in G \operatorname{Pr}(h, \psi(F))$, then $y \in \psi(F)$ such that $h^{n}(y)=\theta_{2}(g, y)$ for some $n \in \mathbb{N}$ and $g_{2} \in G_{2}$. Since $y$ is $G_{2}$ - periodic points of $h$, then $x=\psi^{-1}(y)$ is $G_{1}$ - periodic point of $f$ by Theorem 2.8. Now, we have

$\mathrm{y}=\psi(\mathrm{x}) \notin \mathrm{V} \Rightarrow \theta_{2}\left(\mathrm{~g}_{2}, \mathrm{y}\right) \notin \theta_{2}\left(\mathrm{~g}_{2}, \mathrm{~V}\right)$

$\Rightarrow \mathrm{h}^{\mathrm{n}}(\mathrm{y}) \notin \theta_{2}\left(\mathrm{~g}_{2}, \mathrm{~V}\right)$, for some $\mathrm{n} \in \mathbb{N}$ and $\mathrm{g}_{2} \in \mathrm{G}_{2}$

$\Rightarrow \psi \circ \mathrm{f}^{\mathrm{n}} \circ \psi^{-1}(\psi(\mathrm{x})) \notin \theta_{2}\left(\mathrm{~g}_{2}, \mathrm{~V}\right)$, for some $\mathrm{n} \in \mathbb{N}$ and $\mathrm{g}_{2} \in \mathrm{G}_{2}$

$\Rightarrow \psi\left(\mathrm{f}^{\mathrm{n}}(\mathrm{x})\right) \notin \theta_{2}\left(\mathrm{~g}_{2}, \mathrm{~V}\right)$, for some $\mathrm{n} \in \mathbb{N}$ and $\mathrm{g}_{2} \in \mathrm{G}_{2}$

$\Rightarrow \mathrm{f}^{\mathrm{n}}(\mathrm{x}) \notin \Psi^{-1}\left(\theta_{2}\left(\mathrm{~g}_{2}, \mathrm{~V}\right)\right)$, for some $\mathrm{n} \in \mathbb{N}$ and $\mathrm{g}_{2} \in \mathrm{G}_{2}$

$\Rightarrow \mathrm{f}^{\mathrm{n}}(\mathrm{x}) \notin \theta_{1}\left(\mu^{-1}\left(\mathrm{~g}_{2}\right), \varphi^{-1}(\mathrm{~V})\right)$, for some $\mathrm{n} \in \mathbb{N}$ and $\mathrm{g}_{2} \in \mathrm{G}_{2}$

$\Rightarrow \theta_{1}\left(\mathrm{~g}_{1}, \mathrm{x}\right) \notin \theta_{1}\left(\mathrm{~g}_{1}, \varphi^{-1}(\mathrm{~V})\right)$, for some $\mathrm{n} \in \mathbb{N}$ and $\mathrm{g}_{1}=\mu^{-1}\left(\mathrm{~g}_{2}\right) \in \mathrm{G}_{1}$

$\Rightarrow \mathrm{x} \notin \varphi^{-1}(\mathrm{~V})$, which is a contradiction.

\section{3. $G_{f}-$ orbit of maps}

In this section we state the definitions of orbits and $\mathrm{G}$-orbits of maps and give new properties of $\mathrm{G}$-orbits.

Definition 3.1: [11] Let $X$ be a space and $f: X \rightarrow X$ be a map. We define the orbit of $f$ at a point $x \in X$ as follows $\mathrm{O}(\mathrm{f}, \mathrm{x})=\left\{\mathrm{f}^{\mathrm{n}}(\mathrm{x}): \mathrm{n} \geq 0\right\}$.

Definition 3.2: [6] Let $X$ be a $G$-space and $f: X \rightarrow X$ be a map. We define the $G_{f}-$ orbit of $f$ at a point $x \in X$ as follows $\mathrm{GO}(\mathrm{f}, \mathrm{x})=\left\{\theta\left(\mathrm{g}, \mathrm{f}^{\mathrm{n}}(\mathrm{x})\right): \mathrm{g} \in \mathrm{G}, \mathrm{n} \geq 0\right\}$.

Remark 3.3: Under the trivial action of $G$ on $X$ the notions of orbit and $G_{f}$-orbit are coincided. In the following example we see that $\mathrm{O}(\mathrm{f}, \mathrm{x}) \subseteq \mathrm{GO}(\mathrm{f}, \mathrm{x})$ under a non trivial action of $\mathrm{G}$. 
Example 3.4: Let $X=\{0,1\}, \tau=\{\phi,\{0\}, X\}$ and $G=\{-1,1\}$ under the discrete topology with the action $\theta: G \times X \rightarrow X$ defined by $\theta(-1, x)=1-x, \theta(1, x)=x, x \in X$. Define $f: X \rightarrow X$ by $f(0)=0, f(1)=1$ (the identity map). Then $f$ is continuouson $X$. Then

$\mathrm{GO}(\mathrm{f}, 0)=\{0,1\}, \mathrm{O}(\mathrm{f}, 0)=\{0\}$.

$\mathrm{GO}(f, 1)=\{0,1\}, O(f, 1)=\{1\}$.

Theorem 3.5: Let $X_{i}$ be a $G_{i}$-space, $i=1,2, \ldots, k, F_{i} \in K\left(X_{i}\right)$ and $f_{i}: F_{i} \rightarrow F_{i}$ be a map. If there exists $x_{i} \in F_{i}$ such that $\overline{G_{l} O\left(f_{l}, x_{l}\right)}=F_{i}$, for every $i=1,2, \ldots, k$ then $\overline{G O(f, x)}=F$, where $G=\prod_{i=1}^{k} G_{i}, F=\prod_{i=1}^{k} F_{i}, f=\prod_{i=1}^{k} f_{i}$ and $x=\left(x_{1}, x_{2}, \ldots, x_{k}\right)$.

Proof: First we need to show that $\overline{G O(f, x)} \subseteq F$. If there exists $x_{i} \in F_{i}$ such that $\overline{G_{l} O\left(f_{l}, x_{l}\right)}=F_{i}$, for every $i=$ $1,2, \ldots, k$, then it is easy to see that

$G O(f, x) \subseteq G_{1} O\left(f_{1}, x_{1}\right) \times G_{2} O\left(f_{2}, x_{2}\right) \times \ldots \times G_{k} O\left(f_{k}, x_{k}\right)$.

Hence, $\overline{G O(f, x)} \subseteq \overline{G_{1} O\left(f_{1}, x_{1}\right)} \times \overline{G_{2} O\left(f_{2}, x_{2}\right)} \times \ldots \times \overline{G_{k} O\left(f_{k}, x_{k}\right)}$.

Consequently $\overline{G O(f, x)} \subseteq F$. Now to proof the converse inclusion.Let $y \in F$, then $y=\left(y_{1}, y_{2}, \ldots, y_{k}\right)$, where $x_{i} \in F_{i}$, for $i=1,2, \ldots, k$. Also each $F_{i}$ is such that $\overline{G_{l} O\left(f_{l}, x_{l}\right)}=F_{i}$, for some $x_{i} \in F_{i}$. So there exists a sequence in $G_{i} O\left(f_{i}, x_{i}\right)$ which converges to $y_{i}$, for $i=1,2, \ldots, k$. i.e., there exists a subsequence of for $\left\{\theta_{i}\left(g_{i}, f_{i}^{m}\left(x_{i}\right)\right)\right\}_{m=1}^{\infty}$ which converges to $y_{i}$. So given a neighborhood $V_{y_{i}}$ of $y_{i}$, there exists $N_{i} \in \mathbb{N}$ such that $\forall l \geq N_{i}, \theta_{i}\left(g_{i}, f_{i}^{m}\left(x_{i}\right)\right) \in V_{y_{i}}$. Let $N=$ $\max \left\{N_{i}: i=1,2, \ldots, k\right\}$. If $V$ is a neighborhood of $y$, then $\forall k \geq N, f^{k}(x) \in V$.So $\left\{f^{k}(x)\right\}$ converges to $y$. So $y \in$ $\overline{G O(f, x)}$ and consequently $\in \overline{G O(f, x)}$. Thus we have $\overline{G O(f, x)}=F$ and this completed the proof.

Theorem 3.6: Let $X$ be a $G_{1}$-space,$Y$ be $G_{2}$-space and $f: X \rightarrow X, h: Y \rightarrow Y$ be equivariant topologically conjugate via $(\mu, \psi):\left(G_{1}, X, \theta_{1}\right) \rightarrow\left(G_{2}, Y, \theta_{2}\right)$. Let $F_{1} \in K(X)$. If there exists $x \in F_{1}$ such that $\overline{G_{1} O(f, x)}=F_{1}$, then $\overline{G_{2} O(h, \psi(x))}=\varphi\left(F_{1}\right)$.

Proof: First, note that $\psi\left(F_{1}\right) \in K(Y)$. Suppose, if possible, that $\overline{G_{2} O(h, \psi(x))} \neq \psi\left(F_{1}\right)$.

Then there exists an open $V$ set in $F_{2}=\psi\left(F_{1}\right)$ such that $\overline{G_{2} O(h, \psi(x))} \cap V=\phi$.

Then $\theta_{2}\left(g_{2}, h^{n}(\psi(x)) \notin V\right.$,for every $n \in \mathbb{N}$ and $g_{2} \in G_{2}$. Since $f$ and $h$ are equivariant topologically conjugate via $(\mu, \psi)$, then

$\theta_{2}\left(g_{2}, \psi\left(f^{n}(x)\right) \notin V\right.$,for every $n \in \mathbb{N}$ and $g_{2} \in G_{2}$.

Since the pair $(\mu, \psi)$ is isomorphism, then

$\varphi^{-1}\left(\theta_{2}\left(g_{2}, \psi\left(f^{n}(x)\right)\right)\right) \notin \psi^{-1}(V)$,for every $n \in \mathbb{N}$ and $g_{2} \in G_{2}$.

$\Rightarrow \theta_{1}\left(\mu^{-1}\left(g_{2}\right), f^{n}(x)\right) \notin \psi^{-1}(V)$, for every $n \in \mathbb{N}$ and $g_{2} \in G_{2}$.

then $\theta_{1}\left(g_{1}, f^{n}(x)\right) \notin \psi^{-1}(V)$, for every $n \in \mathbb{N}$ and $g_{1}=\mu^{-1}\left(g_{2}\right) \in G_{1}$,

Which contradicts the fact that $\overline{G_{1} O(f, x)}=F_{1}$. This complete the proof.

\section{G-expansive maps}

In this section we introduce the concept of expansive maps in $G$-space and obtain the necessarily condition under which the map becomes expansive. Further we study some important properties required in Section 5. The following definition is the generalization of the definition of expansive map defined in a metric $G$-space[4]. Here we introduce the definition of expansive map in general topological space ( not necessarily metrizable. Also we show that the two definitions are coincides in a metrizable topological space.

Definition 4.1: [18]If $X$ is a metric $G$-space with metric $d$ and $h \in \mathcal{H}(X)$ then $h$ is called expansive if there exists a $\delta>0$ such that whenever $x, y \in X, x \neq y$ then there exists an integer $n$ satisfying $d\left(h^{n}(x), h^{n}(y)\right)>\delta$.

Definition 4.2.[4]If $X$ is a metric $G$-space with metric $d$ and $h \in \mathcal{H}(X)$ then $h$ is called $G$-expansiveif there exists a $\delta>0$ such that whenever $x, y \in X, G(x) \neq G(y)$ then there exists an integer $n$ satisfying $d\left(h^{n}(u), h^{n}(v)\right)>\delta$, for all $u \in G(x)$ and $v \in G(y)$.

Definition 4.3: If $X$ is a topological space and $h \in \mathcal{H}(X)$ then $h$ is called expansive, if for every $x, y \in X$, with $x \neq y$, then there exist an open set $V$ in $X$, an integer $n \operatorname{such}$ that $h^{n}(x) \in V$ and $h^{n}(y) \notin \bar{V}$.

Definition 4.4.If $X$ is a $G$-space and $h \in \mathcal{H}(X)$ then $h$ is called $G$-expansive, if for every $x, y \in X$, with $G(x) \neq$ $G(y)$, then there exist an open set $V$ in $X$, an integer $n$ such that $f^{n}(u) \in V$ and $f^{n}(v) \notin \bar{V}$, for all $u \in G(x)$ and $v \in G(y)$ 
Remark 4.5: Under the trivial action of $G$ on $X$ the notions of expansive and $G$ - expansive are coincided. It is observed that the notion of expansiveness and the notation of $G$-expansive under a non-trivial action of $G$ are independent of each other.

In the following we shall show that Definition 4.1 and 4.3 are coincides in a metrizable topological space. To this end we state and prove the following lemma.

Lemma 4.6: Let $(X, d)$ be a metric space and let $x, y \in X$ with $x \neq y$, then the following statement are equivalent.

i) (i)There exists $\delta>0$ such that $\mathrm{d}(\mathrm{x}, \mathrm{y})>\delta$.

ii) (ii)There exists an open set $\mathrm{V}$ in $\mathrm{X}$ such that $\mathrm{x} \in \mathrm{Vand} \mathrm{y} \notin \overline{\mathrm{V}}$.

Proof: "(i) $\Rightarrow\left(\right.$ ii)". Suppose (i). Let $V:=B_{\delta}(x)$ be an open ball with center $x$ and radius $\delta$. Then $x \in V$ and $y \notin \bar{V}$. For, if $y \in \bar{V}$, then $d(x, y) \leq \delta$ and this contradicts (i).

"(ii) $\Rightarrow$ (i)". Suppose (ii). Without loss the generality let $V$ be an open ball in $X$ with center $x_{0}$ and radius $r>0$, i.e., $V:=B_{r}\left(x_{0}\right)$ with $x \in V$ and $y \notin \bar{V}$. We have two cases: If $x=x_{0}$, then we can take $\delta=r$. Since $y \notin \bar{V}$, then $d\left(x_{0}, y\right)>\delta$. Consequently $d(x, y)>\delta$.

If $x \neq x_{0}$, then we take $\delta=d\left(x, x_{0}\right)$. Thus

$\delta=d\left(x, x_{0}\right) \leq d(x, y)+d\left(y, x_{0}\right)$.

Since $\notin \bar{V}$, then $d\left(x_{0}, y\right)>0$. Consequently, $d(x, y)>\delta$.

Corollary 4.7: Let $(X, d)$ be a metric space and let $A, B$ be two non-empty disjoint subsets of $X$, then the following statement are equivalent for every $x \in A$ and $y \in B$.

i) There exists $\delta>0$ such that $d(x, y)>\delta$.

ii) There exists an open set $V$ in $X$ such that $x \in V$ and $y \notin \bar{V}$.

Theorem 4.8: If Xis a metrizable topological space, then Definition 4.1 and 4.3 are equivalent.

Proof: Without loss of generality we consider $X$ as a metric space. First, note that if $x, y \in X$ with $x \neq y$ and $h \in \mathcal{H}(X)$, then $h^{n}(x) \neq h^{n}(y)$ and the result follows from Lemma 4.6.

Theorem 4.9: If $X$ is a metrizable topological space, then Definition 4.2 and 4.4 are equivalent.

Proof: The result follows from Corollary 4.5.

Theorem 4.10: Let $X$ be a compact $T_{2} G$-space with a compact group Gand $f \in \mathcal{H}(X)$. If $f^{n}$ not constant for some $n \geq 1$, then $f$ is $G$-expansive on $X$.

Proof: Suppose that $f^{n}$ not constant for some $n \geq 1$. Let $x, y \in X$ with $G(x) \neq G(y)$. Since $f$ is equivariant and oneto-one, then $G(f(x)) \neq G(f(y))$. Since $X$ is $T_{2}$ and $G$ is compact then $G(x), G(y)$ are closed sets in $X$. But $f \in \mathcal{H}(X)$ so $f^{n} \in \mathcal{H}(X)$ and consequently $G\left(f^{n}(x)\right), G\left(f^{n}(y)\right)$ are disjoint closed sets in $X$. Since $X$ is compact and $T_{2}$ then it is normal, thus there exist two disjoint open sets $V$ and $W$ containing $G\left(f^{n}(x)\right), G\left(f^{n}(y)\right)$ respectively such that $\bar{V} \cap \bar{W}=$ $\phi$. Thus we have $f^{n}(x) \in V$ and $f^{n}(y) \notin \bar{V}$. Let $u \in G(x)$ and $v \in G(y)$. Then there exist $g, q \in G$ such that $u=$ $\theta(g, x), v=\theta(q, y)$. Thus we have $f^{n}(u) \in V$ and $f^{n}(v) \notin \bar{V}$ for every $u \in G(x)$ and $v \in G(y)$. This means that $f$ is $G$-expansive.This complete the proof.

Theorem 4.11: Let $X$ be a Urysohn space and $f \in \mathcal{H}(X)$. If $f^{n}$ not constant for some $n \geq 1$, then $f$ is expansive on $X$.

Proof: Let , $y \in X$, with $x \neq y$. Since $f^{n}$ not constant for some $n \geq 1$, then $f^{n}(x) \neq f^{n}(y)$. Since $X$ be a Urysohn space, then there exist two open sets $U, V$ such that $f^{n}(x) \in U, f^{n}(y) \in V$ and $\bar{U} \cap \bar{V}=\phi$. Then $f^{n}(y) \notin \bar{U}$ and consequently $f$ is expansive on $X$.

Lemma4.12: If $\left(G_{1}, X, \theta_{1}\right) \cong_{(\mu, \psi)}\left(G_{2}, Y, \theta_{2}\right)$, then $\psi\left(G_{1}(x)\right)=G_{2}(\psi(x))$.

Proof: We have $G_{1}(x)=\left\{\theta_{1}\left(g_{1}, x\right): g_{1} \in G_{1}\right\}$. Then

$\psi\left(G_{1}(x)\right)=\left\{\psi\left(\theta_{1}\left(g_{1}, x\right)\right): g_{1} \in G_{1}\right\}$.

Since $\left(G_{1}, X, \theta_{1}\right) \cong(\mu, \psi)\left(G_{2}, Y, \theta_{2}\right)$, then

$\left.\psi\left(G_{1}(x)\right)=\left\{\theta_{2}\left(\mu\left(g_{1}\right), \psi(x)\right)\right): g_{1} \in G_{1}\right\}$

$\left.=\left\{\theta_{2}\left(g_{2}, \psi(x)\right)\right): g_{2} \in G_{2}\right\}=G_{2}(\psi(x))$.

Then the proof is complete. 
Theorem4.13: Let $X$ be a $G_{1}$-space , $Y$ be $G_{2}$-space and $h_{1} \in \mathcal{H}(X), h_{2} \in \mathcal{H}(Y)$ be equivariant topologically conjugate via

$(\mu, \psi):\left(G_{1}, X, \theta_{1}\right) \rightarrow\left(G_{2}, Y, \theta_{2}\right)$.

If $h_{1}$ is $G_{1}$-expansive, then $h_{2}$ is $G_{2}$-expansive.

Proof: Suppose that $h_{1}$ is $G_{1}$ - expansive. Let $y_{1}, y_{2} \in Y$, with $G_{2}\left(y_{1}\right) \neq G_{2}\left(y_{2}\right)$. Since $\varphi$ is bijective then there exists $x_{1}, x_{2} \in X$ such that $y_{1}=\varphi\left(x_{1}\right), y_{2}=\varphi\left(x_{2}\right)$ and this implies that $x_{1}=\varphi^{-1}\left(y_{1}\right), x_{2}=\varphi^{-1}\left(y_{2}\right)$. Since $\varphi$ is equivariant, then so is $\varphi^{-1}$ and consequently $\varphi^{-1}$ is pseudoequivariant. Therefore by Lemma 4.4 we have $\varphi^{-1}\left(G_{2}\left(y_{1}\right)\right)=G_{1}\left(\varphi^{-1}\left(y_{1}\right)\right)=G_{1}\left(x_{1}\right)$.

Similarly $\varphi^{-1}\left(G_{2}\left(y_{2}\right)\right)=G_{1}\left(\varphi^{-1}\left(y_{2}\right)\right)=G_{1}\left(x_{2}\right)$. Since $G_{2}\left(y_{1}\right) \neq G_{2}\left(y_{2}\right)$ and $\varphi$ is bijective, then $G_{1}\left(x_{1}\right) \neq G_{1}\left(x_{2}\right)$. By hypothesis there exist open set $V_{1}$ in $X, n \in \mathbb{N}$, such that $h_{1}^{n}\left(u_{1}\right) \in V_{1}$ and $h_{1}^{n}\left(u_{2}\right) \notin \bar{V}_{1}$, for all $u_{1} \in G_{1}\left(x_{1}\right)$ and $u_{2} \in G_{1}\left(x_{2}\right)$.Hence $\varphi\left(h_{1}^{n}\left(u_{1}\right)\right) \in \varphi\left(V_{1}\right)$ and $\varphi\left(h_{1}^{n}\left(u_{2}\right)\right) \notin \varphi\left(\overline{V_{1}}\right)$, for all $u_{1} \in G_{1}\left(x_{1}\right)$ and $u_{2} \in G_{1}\left(x_{2}\right)$. This implies that $h_{2}^{n}\left(\varphi\left(u_{1}\right)\right) \in \varphi\left(V_{1}\right)$ and $h_{2}^{n}\left(\varphi\left(u_{2}\right)\right) \notin \varphi\left(\bar{V}_{1}\right)$, for all $u_{1} \in G_{1}\left(x_{1}\right)$ and $u_{2} \in G_{1}\left(x_{2}\right)$.Set $V_{2}=\varphi\left(V_{1}\right)$. Since $\varphi$ is homeomorphism then $V_{2}$ is open and $\varphi\left(\overline{V_{1}}\right)=\overline{\varphi\left(V_{1}\right)}=\overline{V_{2}}$. Let $v_{1} \in G_{2}\left(y_{1}\right), v_{2} \in G_{2}\left(y_{2}\right)$, then

$\varphi^{-1}\left(v_{1}\right) \in \varphi^{-1}\left(G_{2}\left(y_{1}\right)\right)$, and $\varphi^{-1}\left(v_{2}\right) \in \varphi^{-1}\left(G_{2}\left(y_{2}\right)\right)$

Thus

$\varphi^{-1}\left(v_{1}\right) \in G_{1}\left(\varphi^{-1}\left(y_{1}\right)\right)=G_{1}\left(x_{1}\right), \varphi^{-1}\left(v_{2}\right) \in G_{1}\left(\varphi^{-1}\left(y_{2}\right)\right)=G_{1}\left(x_{2}\right)$.

Consequently, $h_{2}^{n}\left(\varphi\left(\varphi^{-1}\left(v_{1}\right)\right) \in V_{2}\right.$ and $h_{2}^{n}\left(\varphi\left(\varphi^{-1}\left(v_{2}\right)\right) \notin \bar{V}_{2}\right.$.i.e., $h_{2}^{n}\left(v_{1}\right) \in V_{2}$ and $h_{2}^{n}\left(v_{2}\right) \notin \overline{V_{2}}$.This mean that $h_{2}$ is $G_{2}$ - expansive.

Corollary 4.14: Let $X, Y$ be two topological spaces and $h_{1} \in \mathcal{H}(X), h_{2} \in \mathcal{H}(Y)$ be topologically conjugate via $: X \rightarrow Y$. If $h_{1}$ is expansive, then so is $h_{2}$.

Theorem 4.15: Let $X$ be a $G_{1}$-space,$Y$ be $G_{2}$-space and $h_{1} \in \mathcal{H}(X), h_{2} \in \mathcal{H}(Y)$. If either $h_{1}$ is $G_{1}$-expansive or $h_{2}$ is $G_{2}$-expansive, then $h_{1} \times h_{2} \in \mathcal{H}(X \times Y)$ is $G_{1} \times G_{2}$-expansive.

Proof: First, note the product of two homeomorphism maps is also homeomorphism, thus the statement $h_{1} \times h_{2} \in$ $\mathcal{H}(X \times Y)$ is true. Suppose that $h_{1}$ is $G_{1}$-expansive. Let $z=\left(x_{1}, y_{1}\right), w=\left(x_{2}, y_{2}\right) \in X \times Y$, with $G(z) \neq G(w)$. That is $G_{1} \times G_{2}\left(x_{1}, y_{1}\right) \neq G_{1} \times G_{2}\left(x_{2}, y_{2}\right)$ or $G_{1}\left(x_{1}\right) \times G_{2}\left(y_{1}\right) \neq G_{1}\left(x_{2}\right) \times G_{2}\left(y_{2}\right)$. Then either $G_{1}\left(x_{1}\right) \neq G_{1}\left(x_{2}\right)$ or $G_{2}\left(y_{1}\right) \neq$ $G_{2}\left(y_{2}\right)$. Suppose that $G_{1}\left(x_{1}\right) \neq G_{1}\left(x_{2}\right)$. Since $h_{1}$ is $G_{1}$-expansive, then there exist an open set $V_{1}$ and an integer $n$ such that $h_{1}^{n}\left(u_{1}\right) \in V_{1}$ and $h_{1}^{n}\left(u_{2}\right) \notin \bar{V}_{1}$, for all $u_{1} \in G_{1}\left(x_{1}\right)$ and $u_{2} \in G_{1}\left(x_{2}\right)$.Set $V=V_{1} \times Y$, then $V$ is an open set in $X \times Y$. Now

$\left(h_{1} \times h_{2}\right)^{n}(z)=h_{1}^{n} \times h_{2}^{n}(z)=\left(h_{1}^{n}\left(x_{1}\right), h_{2}^{n}\left(y_{1}\right)\right) \in V_{1} \times Y=V$ and

$\left(h_{1} \times h_{2}\right)^{n}(w)=h_{1}^{n} \times h_{2}^{n}(w)=\left(h_{1}^{n}\left(x_{2}\right), h_{2}^{n}\left(y_{2}\right)\right) \in \bar{V}_{1} \times Y=\bar{V}$.

This means that $h_{1} \times h_{2} \in \mathcal{H}(X \times Y)$ is $G_{1} \times G_{2}$-expansive.

Corollary 4.16. Let $X$ be two topological spaces and $h_{1} \in \mathcal{H}(X), h_{2} \in \mathcal{H}(Y)$. If either $h_{1}$ or $h_{2}$ is expansive, then so is $h_{1} \times h_{2} \in \mathcal{H}(X \times Y)$.

\section{G-expansive chaotic maps}

This section is the main section in this paper. Here we introduce a new definition of chaotic maps called $G$-expansive chaotic maps. Also we give some new properties of such maps. Through this section we shall show that our definition has no redundant.

Definition 5.1: Let $X$ be a $G-$ space and $F$ be a compact subset of $X$. A continuous map $f: X \rightarrow X$ is said to be $G$-expansive chaotic on $F$ if

i) $\overline{G O(f, x)}=F$, for some $x \in F$,

ii) $\overline{G P_{r}(X)}=F$, and

iii) $f$ is $G$-expansive on $F$.

\section{Notation 5.2:}

i) $\quad E G C(F)=\{f: F \rightarrow F: f$ is expansive $G-$ chaotic on $F\}$.

ii) $E G C H(X)=\{F \in K(X): E G C(F) \neq \phi\}$.

Definition5.3: $\mathrm{A} G$-space $X$ is said to be expansive $G$-chaos space if $\operatorname{EGCH}(X) \neq \phi$. In this case the elements of $E G C H(X)$ are called expansive $G$ - chaotic sets.

In the following examples we shall show that the statements (i),(ii) and (iii) in Definition 6.1 are independent and not two of them imply the other. That is there are not redundant hypothesis in our definition of chaos. 
Example 5.4: In this example we shall show that (i) and (ii) $\nRightarrow$ (iii). Let $X=\{0,1\}, \tau=\{\phi,\{0\}, X\}$ and $G=\{-1,1\}$ under the discrete topology with the action $\theta: G \times X \rightarrow X$ defined by $\theta(-1, x)=1-x, \theta(1, x)=x, x \in X$. Define $f: X \rightarrow X$ by $f(0)=0, f(1)=1$ (the identity map). Then $f$ is continuous on $X$. It is easy to see that

a. $\overline{G O(f, 0)}=X$,

b. $\overline{\operatorname{GPr}(f, X)}=X$, but

c. $f$ is not $G$-expansive on $X$.

Example 5.5: In this example we shall show that (ii) and (iii) $\nRightarrow(\mathrm{i})$. Let $X=[0,1]$ under the usual topology and $G=\{-1,1\}$ under the discrete topology with the action $\theta: G \times X \rightarrow X$ defined by $\theta(-1, x)=1-x, \theta(1, x)=x$, $x \in X$. Define $f: X \rightarrow X$ by $f(x)=x$ (the identity map). Then $f$ is continuous on $X$. It is easy to see that

a. $\overline{G O(f, x)} \neq X$, for all $x \in X$.

b. $\overline{\operatorname{GPr}(f, X)}=X$.

c. $f$ is $G$-expansive on $X$.

Example 5.6: In this example we shall show that(i) and(iii) $\nRightarrow(i i)$ Let $X=[0,3 / 4]$ under the usual topology and $G=\{-1,1\}$ under the discrete topology with the action $\theta: G \times X \rightarrow X$ defined by $\theta(-1, x)=1-x, \theta(1, x)=x$, $x \in X$. Define $f: X \rightarrow X$ by

$f(x)=\left\{\begin{array}{cc}\frac{3}{2} x, & \text { if } 0 \leq x \leq 1 / 2 \\ \frac{3}{2}(1-x), & \text { if } 1 / 2 \leq x \leq 3 / 4\end{array}\right.$.

Then $f$ is homeomorphism on $X$. It is easy to see that

a. $\overline{G O(f, x)}=X$, for all $x \in X$.

b. $\overline{\operatorname{GPr}(f, X)} \neq X$.

c. $f$ is $G$-expansive on $X$.

Example 5.7: In this example we shall show that (iii) $\nRightarrow($ ii) and (i) Let $X=[0,1]$ under the usual topology and $G=$ $\{-1,1\}$ under the discrete topology with the action $\theta: G \times X \rightarrow X$ defined by $\theta(-1, x)=1-x, \theta(1, x)=x, x \in X$. Define $f: X \rightarrow X$ by $f(x)=x^{2}$. Then $f$ is homeomorphism on $X$. It is easy to see that

a. $\overline{G O(f, x)} \neq X$, for all $x \in X$.

b. $\overline{\operatorname{GPr}(f, X)} \neq X$.

c. $f$ is $G$-expansive on $X$.

Theorem 5.7: Let $b\left(G_{1}, X, \theta_{1}\right) \cong{ }_{(\mu, \psi)}\left(G_{2}, Y, \theta_{2}\right)$. If $X$ is expansive $G_{1}-$ chaos space, then $Y$ is expansive $G_{2}-$ chaos space.

Proof: This follows from Theorems 2.9, 3.6 and 4.13.

Theorem 5.8: Let $X$ be a $G_{1}$-space, $Y$ be a $G_{2}-$ space and $f_{1}: X_{1} \rightarrow X_{1}, f_{2}: X_{2} \rightarrow X_{2}$ be equivariant maps. If $f_{1}$ is expansive $G_{1}$-chaotic on $F_{1} \in K\left(X_{1}\right)$ and $f_{2}$ is expansive $G_{2}$ - chaotic $F_{2} \in K\left(X_{2}\right)$, then $f=f_{1} \times f_{2}$ is expansive $G_{1} \times$ $G_{2}$ - chaotic on $F=F_{1} \times F_{2} \in K\left(X_{1} \times X_{2}\right)$.

Proof: This follows from Theorems 2.7, 3.5 and 4.15.

Corollary 5.9 Let $X_{i}$ be a $G_{i}$-space, $i=1,2, \ldots, k$ and $f_{i}: X_{i} \rightarrow X_{i}$, be paire wise equivariant maps. If $f_{i}$ is expansive $G_{i}$ - chaotic on $F_{i} \in K\left(X_{i}\right), i=1,2, \ldots, \mathrm{k}$, then $\mathrm{f}$ is expansive $\mathrm{G}$ - chaotic on $\mathrm{F} \in \mathrm{K}(\mathrm{X})$ wherer $\mathrm{X}=\prod_{\mathrm{i}=1}^{\mathrm{k}} \mathrm{X}_{\mathrm{i}}, \quad, \mathrm{G}=$ $\prod_{i=1}^{k} G_{i}, F=\prod_{i=1}^{k} F_{i}$, and $f=\prod_{i=1}^{k} f_{i}$.

\section{Examples of expansive chaotic maps}

In this final section we give some examples of maps that are G -expansive chaotic or not G-expansive chaotic.

Example 6.1: Let $X=[0,1]$ with theusual topology and let $\mathbb{Z}_{2}$ act on $X$ by $\theta(0, x)=x$ and $\theta(1, x)=1-x, x \in X$.

Consider $\mathrm{f}: \mathrm{X} \rightarrow \mathrm{X}$ defined by 
$f(x)=\left\{\begin{array}{cc}x+\frac{1}{3}, & 0 \leq x \leq \frac{2}{3} \\ 1-9\left(x-\frac{2}{3}\right), & \frac{2}{3}<x<\frac{23}{30} \\ x-\frac{2}{3}, & \frac{23}{30} \leq x \leq 1\end{array}\right.$

Clearly $\mathrm{f}$ is $\mathbb{Z}_{2}$-expansive chaotic.

Example 6.2: $\operatorname{Let} X=[0,1]$ with theusual topology and let $\mathbb{Z}_{2}$ act on $X$ by $\theta(0, x)=x$ and $\theta(1, x)=1-x, x \in X$. Consider the usual tent map $\mathrm{T}: \mathrm{X} \rightarrow \mathrm{X}$ defined by

$\mathrm{T}(\mathrm{x})=\left\{\begin{array}{cc}2 \mathrm{x}, & 0 \leq \mathrm{x} \leq \frac{1}{2} \\ 2(1-\mathrm{x}), & \frac{1}{2} \leq \mathrm{x} \leq 1\end{array}\right.$

Since there are points $\mathrm{x} \neq \mathrm{y}$ such that $\mathrm{T}(\theta(\mathrm{g}, \mathrm{x}))=\mathrm{T}(\theta(\mathrm{g}, \mathrm{y}))$

For some $g \in \mathbb{Z}_{2}$ thenT is not $\mathbb{Z}_{2}$-expansive map. Thus it is not $\mathbb{Z}_{2}$-expan-sive chaotic.

Example 6.3: From Examples 1.4 and 6.2 we conclude that the logistic map $Q_{4}$ is not $\mathbb{Z}_{2}$-expansive map and hence it is not $\mathbb{Z}_{2}-$ expansive chaotic.

\section{References}

[1] Aoki, N. and Hiraide, K. Topological Theory of Dynamical Systems. , North Holland Mathematical Library, North-Holland, Amsterdam (1994).

[2] Arhangel'skii, A. and Tkachenko, M. Topological Groups and Related Structures, Atlantis Press, World Scientific, (2008).

[3] Choi, T.and Kim, J. Decomposition Theorem on G-Spaces. , Osaka Journal of the Indian Mathematical, 46(2009), no.1, 87-104.

[4] Das, R.Expansive self-homeomorphism on G -Spaces., Periodica Math.Hungarica, 31(1995), 123-130.

[5] Das, R. .On G-Expansive Homeomorphisms and Generators, the Journalof the Indian Mathematical Society. New Series, 72(2005), no., 1-4, 83-89.

[6] Das, R. And Das, T. .Topological Transitive of Uniform Limit Function on G-Spaces, The Journal of Mathematical Analysis, 6 (2012), no.29-32, 1491-1499.

[7] Das, R. and Das, T.Chaos of Product Map on G -Spaces, Int. Journal of Math. Forum, 8(2013), no.13, 647-652

[8] Das, R. and Das, T. .A note on Representation of Pseudovariant Maps., Math. Solvaca 62(2012).no.1, 137-142.

[9] Das, R. and Das, T.On Properties of G-Expansive Homeomorpisms. , Math. Solvaca62 (2012).no.3, 531-538.

[10] Das, R. and Das, T.On Extension of G-Expansive Homeomorpisms, the Journal of the Indian Mathematical Society. New Series, 67(2000), no. 1-4, 35-41.

[11] Devaney, R.L. An Introduction to Chaotic Dynamical Systems. , Addison-Wesley, 1989.

[12] Gottschalk,W. and Hedlund,G. .Topological Dynamics. , Amer, Math, Soci(1955) .

[13] Husain, T.Introduction toTopological groups., W.B. Saunders Company Philadelphia And London (1966) .

[14] Kumar, V.On Chaotic and Fractals in General Topological Spaces., P.H.D. Cochin University of Science and Technology under Faculty of Science. 2001.

[15] Richeson, D. and Wiseman, J.Positive Expansive Homeomorphisms of Compact Spaces, IJMS 54(2004), 2907-2910.

[16] Shah,E. and Das, T. .Consequence of Shadowing Property ofG-Spaces. ,Int.Journal of Math. Analysis, 7(2013), no.12, 579-588.

[17] Shah, E. and Das, T.On Pseudo Orbit tracing Property of G -Spaces., JP Journal of Geometry and Topology, 3(2003), no.2, 101-112.

[18] Utz,, W.R. .Unstable Homeomorphism. Proceedings of the American Mathematical Society, 1(1950), 769-774.

[19] Willard, S. General Topology. , Addison-Westy Pub.co, Inc.1970. 